\title{
Extracellular Vesicles from Adipose Tissue-A Potential Role in Obesity and Type 2 Diabetes?
}

\author{
Xuan Gao', Carlos Salomon ${ }^{2,3,4,5}$ and Dilys J. Freeman ${ }^{1 *}$ \\ 1 Institute of Cardiovascular and Medical Sciences, University of Glasgow, Glasgow, United Kingdom, ${ }^{2}$ Exosome Biology \\ Laboratory, Centre for Clinical Diagnostics, University of Queensland Centre for Clinical Research, Royal Brisbane and \\ Women's Hospital, The University of Queensland, Brisbane, QLD, Australia, ${ }^{3}$ Faculty of Pharmacy, Department of Clinical \\ Biochemistry and Immunology, University of Concepción, Concepción, Chile, ${ }^{4}$ Maternal-Fetal Medicine, Department of \\ Obstetrics and Gynecology, Ochsner Clinic Foundation, New Orleans, LA, United States, ${ }^{5}$ Mater Research Institute- \\ University of Queensland, Translational Research Institute, Woolloongabba, QLD, Australia
}

OPEN ACCESS

Edited by:

Francisco Gracia-Navarro, University of Cordoba, Spain

Reviewed by:

Edward Owen List,

Ohio University,

United States

María Pardo,

Servicio Gallego de Salud, Spain

${ }^{*}$ Correspondence:

Dilys J. Freeman

dilys.freeman@glasgow.ac.uk

Specialty section: This article was submitted to Systems and

Translational Endocrinology, a section of the journal

Frontiers in Endocrinology

Received: 31 May 2017 Accepted: 02 August 2017 Published: 18 August 2017

Citation:

Gao X, Salomon C and Freeman DJ (2017) Extracellular Vesicles from Adipose Tissue-A Potential Role in

Obesity and Type 2 Diabetes?

Front. Endocrinol. 8:202. doi: 10.3389/fendo.2017.00202
Adipose tissue plays a key role in the development of insulin resistance and its pathological sequelae, such as type 2 diabetes and non-alcoholic fatty liver disease. Dysfunction in the adipose tissue response to storing excess fatty acids as triglyceride can lead to adipose tissue inflammation and spillover of fatty acids from this tissue and accumulation of fatty acids as lipid droplets in ectopic sites, such as liver and muscle. Extracellular vesicles (EVs) are released from adipocytes and have been proposed to be involved in adipocyte/macrophage cross talk and to affect insulin signaling and transforming growth factor $\beta$ expression in liver cells leading to metabolic disease. Furthermore EV produced by adipose tissue-derived mesenchymal stem cells (ADSC) can promote angiogenesis and cancer cell migration and have neuroprotective and neuroregenerative properties. ADSC EVs have therapeutic potential in vascular and neurodegenerative disease and may also be used to target specific functional miRNAs to cells. Obesity is associated with an increase in adipose-derived EV which may be related to the metabolic complications of obesity. In this review, we discuss our current knowledge of EV produced by adipose tissue and the potential impact of adipose tissue-derived EV on metabolic diseases associated with obesity.

Keywords: adipocytes, extracellular vesicles, adipose-derived mesenchymal stem cells, differentiation, insulin resistance, non-alcoholic fatty liver disease

\section{INTRODUCTION}

Over the last few decades adipose tissue and adipocyte function has been under extensive study due to their central role in energy homeostasis, obesity, and diabetes $(1,2)$. The discovery of adipokines has led to the recognition of the key role of adipose tissue secretory products in mediating the consequences of excess adipose tissue accumulation and its wider role in metabolism. More recently, adipose tissue secretion of extracellular vesicles (EVs) and their potential role in the regulation of metabolism and the development of insulin resistance (IR) and type 2 diabetes has come under investigation. This article will review the data available on the nature of the EV produced by the component parts of adipose tissue and their potential local and remote end-organ effects.

Extracellular vesicles are spherical vesicles with an outer lipid bilayer which are released from almost all living cells from bacteria to multicellular organisms (3). EVs are classified according to their size and the pathway by which they were produced (i.e., endocytic or plasma membrane). EVs are grouped by size and origin as exosomes $(\sim 40-100 \mathrm{~nm})$, microvesicles $(\sim 100-1,000 \mathrm{~nm})$, and 
apoptotic bodies ( 1,000-5,000 nm). Microvesicles and apoptotic bodies are formed directly via blebbing of the plasma membrane, whereas exosomes are produced via an endocytic pathway (4). It is difficult to classify EV as there is currently a lack of specific EV markers and there are no internationally accepted definitions of EV. EV may be isolated by a variety of different methods, the most common being differential and buoyant density gradient ultracentrifugation, gel filtration chromatography or other size separation techniques, flow cytometry, or by precipitation using polymers or antibodies (5). EVs play an important role in intercellular communications. With a wide range of inhibitory and stimulatory effects, EV can influence a variety of cell functions, including cytokine production, cell proliferation, apoptosis, and metabolism (6). These effects are mediated by the content of EV including RNA (mRNA, miRNA, and other RNAs), protein, and lipids (3). The distribution of EV is widespread, and EV can be isolated both in vivo (mainly from body fluids, such as plasma/ serum, urine, cerebrospinal fluid, saliva, etc.) and in vitro (from cell-conditioned media). The composition and function of EV derived from adipose tissue is poorly understood but of major interest due to the central role of obesity in type 2 diabetes mellitus (T2DM).

\section{ADIPOSE TISSUE STRUCTURE AND FUNCTION}

\section{Types of Adipose Tissue}

Human white adipose tissue is distributed throughout the body with the main depots classified as subcutaneous adipose tissue and visceral adipose tissue (7). Eighty percent of white adipose tissue is located in the subcutaneous compartment and up to $10-20 \%$ is located in the visceral compartment, mainly around the mesentery and omentum (7). There are also small quantities of adipose tissue located around blood vessels (perivascular adipose tissue) and in liver, muscle, joints, and bone marrow. While subcutaneous adipose tissue functions predominately benignly as a storage depot for excess fatty acids, visceral adipose tissue is more closely linked to the adverse metabolic and inflammatory profile observed in individuals with obesity and IR (8-10). Brown adipose tissue stores are substantial in rodents but in humans are mainly only found in infants or in adults who have undergone cold adaptation (11). Brown adipose tissue promotes non-shivering thermogenesis via the expression of uncoupling protein 1 in its mitochondrial membranes and may have an important role in energy homeostasis (12). White adipose tissue can be induced to express some of the features of brown adipose tissue and the resultant adipocytes are termed beige (13).

\section{Cellular Composition of Adipose Tissue}

Adipose tissue comprises adipocytes and adipose-derived stromal cells (Figure 1). Adipocytes are the main cell type in adipose tissue. Excess calories, as fatty acids, are stored in lipid droplets within adipocytes in the form of triglyceride. In the postprandial period, newly formed, smaller adipocytes more avidly take up free fatty acids released from circulating triglyceride in plasma lipoproteins by lipoprotein lipase resulting in the production of larger adipocytes (14). Adipose stromal cells comprise pre-adipocytes, endothelial cells, fibroblasts, lymphocytes, macrophages, myeloid cells, pericytes, smooth muscle cells, and mesenchymal stromal stem cells (15). Adipose stromal cells support the proliferation and the differentiation of pre-adipocytes to adipocytes in vivo and in vitro and secrete a variety of cytokines and growth factors exerting potential paracrine effects (15).

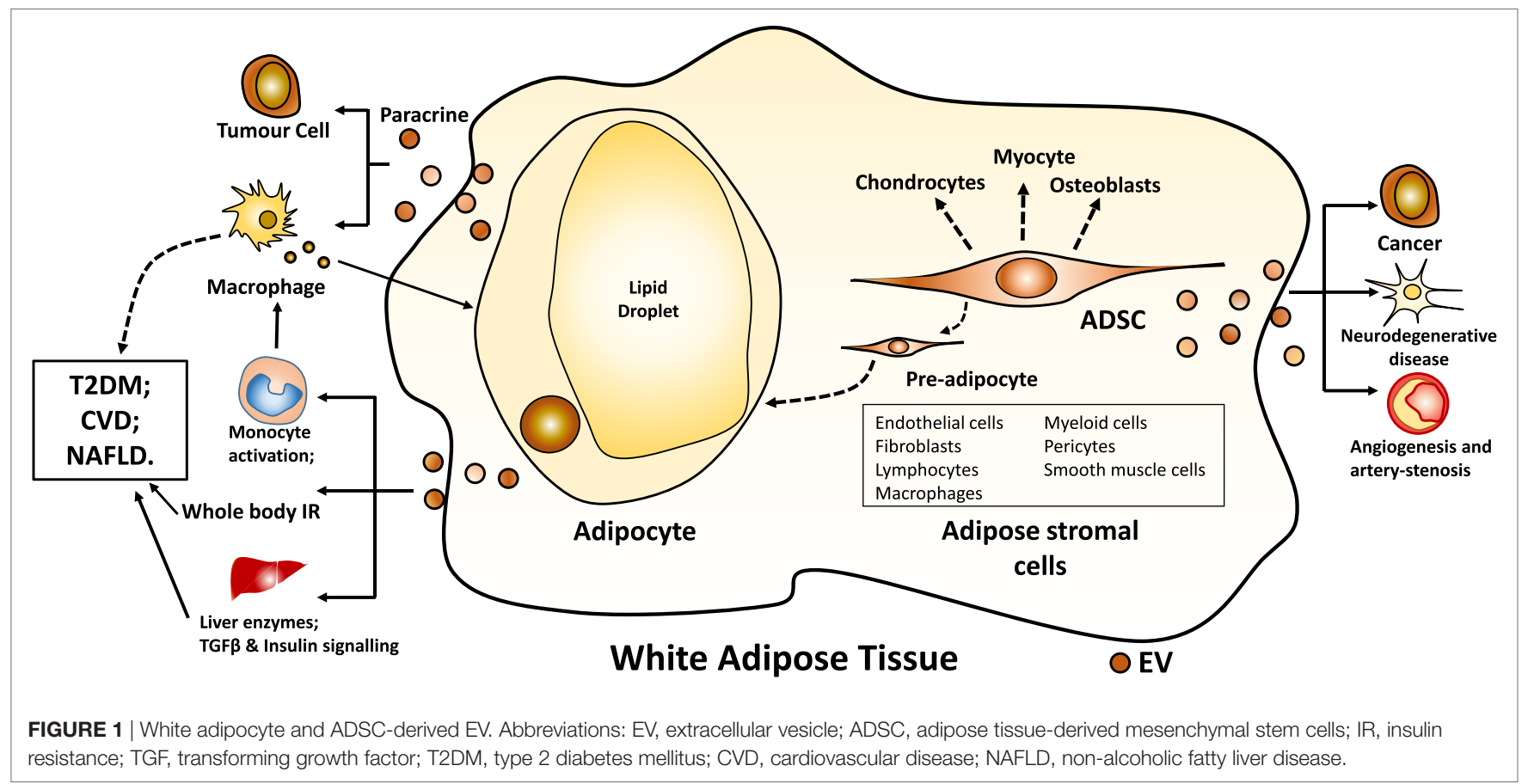


adipose tissue-derived mesenchymal stem cells (ADSC) are multipotent and can differentiate into adipocytes, osteoblasts, chondrocytes, and myocytes (16).

\section{Adipose Tissue Expansion}

When individuals become obese, their excess calorific intake is stored in the form of triglycerides within the adipocytes of white adipose tissue. If there is insufficient capacity in mature adipocytes, new adipocytes are formed from pre-adipocytes in order to increase storage capacity (17). The formation of adipocytes (adipogenesis) occurs in two phases (18). The first phase of white adipose tissue adipogenesis is committed to differentiation and involves the production of committed white pre-adipocytes from mesenchymal stem cells. Once committed, pre-adipocytes lose their multi-potency and can only differentiate into adipocytes or proliferate. Terminal differentiation to form mature white adipocytes results in the characteristic appearance of the mature adipocyte containing one single lipid droplet that occupies almost all the space within the cell. Similarly, brown adipose tissue differentiation comprises a committed differentiation step followed by a terminal differentiation step (18).

In some individuals, there appears to be a limited ability to produce mature adipocytes from pre-adipocytes (hyperplasic adipocyte expansion) and instead excess fatty acids are stored in existing mature adipocytes leading to an increase in their size (hypertrophic expansion) (19). Larger adipocytes tend to be more dysfunctional, and they become insulin resistant resulting in increased lipolysis due to resistance to the anti-lipolytic effects of insulin (8). Failure of angiogenesis and provision of an adequate blood supply to hypertrophic adipocytes leads to necrosis, macrophage infiltration into adipose tissue and inflammation and adipokine release. "Spillover" of fatty acids unable to be retained in subcutaneous adipocytes leads to an increase in the visceral fat compartment and eventually flux of fatty acids into ectopic sites, stored as intracellular lipid droplets in tissues, such as liver and the pancreas. The formation of ectopic fat is closely linked to the development of IR and T2DM, and individuals with limited adipocyte expandability, such as South Asians, are at increased risk of type 2 diabetes (20).

\section{EVs FROM ADIPOCYTES}

\section{Composition of Adipocyte EVs}

Extracellular vesicle production has been studied in whole adipose tissue explants (subcutaneous and visceral) $(21,22)$, isolated adipocytes, in vitro differentiated adipocytes (21) and in ADSC (see later). Kranendonk et al. (21) characterized EV isolated by differential ultracentrifugation from ex vivo subcutaneous and visceral adipose tissue explant cultures and from human adipocytes differentiated in vitro from Simpson Golabi Behmel Syndrome (SGBS) pre-adipocytes (23). Both adiponectin-positive and adiponectin-negative EVs were produced by whole adipose tissue, as determined by flow cytometry and could be separated by differential ultracentrifugation (21). The authors suggested that adiponectin-positive EV came specifically from adipocytes, rather than stromal cells (21). Differentiated human adipocytes produced EV containing the presumed adipose-specific markers FABP-4 and adiponectin as well as a number of inflammatory adipokines, including MIF, TNF $\alpha$, MCSF, and RBP-4 (21). Adipokine profile differed between subcutaneous adipose tissue $\mathrm{EV}$ and visceral adipose tissue EV with concentrations of IL-6, MIF, and MCP-1 significantly higher in visceral adipose tissue $\mathrm{EV}$ compared to those from subcutaneous adipose tissue (24). The exosome miRNA profile from lean and obese individuals was compared and 55 differentially expressed miRNA were found (25). The differentially expressed miRNAs identified were predicted to regulate the transforming growth factor (TGF) $\beta$-signaling and wnt/ $\beta$-catenin signaling pathways (25). A differential EV proteomic profile has also been observed between obese diabetic and obese non-diabetic rats (26).

Extracellular vesicle production by adipocytes has also been studied in the mouse 3T3-L1 pre-adipocyte cell line which can be differentiated to mature adipocytes (27). EV concentration across a size range of $0-1,000 \mathrm{~nm}$ was measured by nano-tracking analysis and concentrations of all particle sizes were more than threefold higher prior to adipogenesis (27). Higher particle concentrations were particularly observed for particles less than $300 \mathrm{~nm}$. Lipid composition of EV was different after adipogenesis with higher membrane phosphatidyl serine content and a higher proportion of arachidonic acid (27). There was no change in EV protein marker expression (CD9, CD63, TSG101, and Alix) or PPAR $\gamma$ protein content after differentiation but FABP-4 and PREF-1 levels decreased while adiponectin content almost doubled (27). 3T3-L1 adipocyte exocytosis has been shown to be stimulated by cAMP via a PKA-independent pathway and this was regulated via both calcium-dependent and -independent processes (28). Exosome release from 3T3-L1 cells was increased by the long chain omega-3 fatty acid docosahexaenoic acid (29). Because of potential hypoxia in adipose tissue resulting from adipocyte hypertrophy, the effect of hypoxia on 3T3-L1 differentiated adipocytes exosome production was assessed (30). 3T3-L1 adipocytes exposed to hypoxia had a different exosome proteomic profile compared to control adipocyte exosomes (30). In particular, exosomes produced under hypoxic conditions were enriched in enzymes involved in de novo lipogenesis and these exosomes were able to promote lipid accumulation in recipient 3T3-L1 cells.

Murine brown adipocytes produce exosomes and their production is increased by cAMP treatment (cAMP is the second messenger induced by cold exposure and $\beta$-adrenergic stimulation) (31). Beige, but not white, adipocyte exosome production was increased nearly 11 -fold by treatment with cAMP (31). When mouse whole adipose tissue was exposed to cold, exosome production was also increased (31). miRNA profiling of the exosomes produced by murine brown adipocytes identified miR-92a as a specific marker that was downregulated on cold exposure (31). Feeding mice a high fat diet induced whitening of brown adipose tissue and an increase in serum miR-92a levels, whereas cold exposure was associated with decreased serum miR-92a levels. In lean humans whose brown adipose tissue activity was assessed by labeled glucose uptake, there was a negative correlation between brown adipose tissue activity and serum miR-92a levels (31). Furthermore, in 10 human subjects exposed 
to cold acclimatization for 10 days, serum miR-92a abundance was decreased and the change in miR-92a levels correlated with the change in brown adipose tissue activity.

\section{Functions of Adipose Tissue-Derived EV}

Extracellular vesicles derived from adipose tissue may play a role in the paracrine cross talk between adipocytes and macrophages (Figure 1). EVs secreted from both subcutaneous and visceral adipose tissue explants and from SGBS-derived adipocytes were able to promote the differentiation of primary monocytes into macrophages. These macrophages had the same cytokine secretory profile as macrophages found in human adipose tissue, reflecting a mixed M1/M2 phenotype (21). Adiponectin-positive EVs were much more effective than adiponectin-negative EVs in effecting monocyte differentiation as were EVs derived from visceral, rather than subcutaneous tissue explants (21). Conditioned medium was collected from macrophages exposed to adipose tissue EV. When human adipocytes were then exposed to this conditioned macrophage medium, insulin signaling as assessed by Akt phosphorylation was inhibited in the adipocytes (21). Differentiated 3T3-L1 adipocytes when stressed by exposure to palmitic acid produced microparticles which could act as chemoattractants for monocytes and primary macrophages (32). Purified murine adipose tissue exosome-like vesicles when injected back into mice were taken up by peripheral blood monocytes which then differentiated into macrophages secreting TNF $\alpha$ and IL-6 (33). When exosome-like vesicles produced by ob/ob mice, which have a high RBP-4 content, were injected into wild-type mice, the recipient mice developed IR (33). This response was less marked in TLR4 knockout mice (33). Taken together these data provide evidence that EV produced by adipocytes may communicate with immune cells and influence whole body IR.

Kranendonk et al. looked at the effects of subcutaneous and visceral adipose tissue EV on insulin signaling and expression of genes involved in gluconeogenesis in a hepatocyte cell line (HepG2) and in myotubes differentiated from a myoblast cell line (C2C12) (24). There was a mixed response to individual adipose tissue EV preparations in hepatocytes. Adipose tissue EV from the majority of individuals inhibited Akt phosphorylation while adipose tissue EVs from a few individuals stimulated Akt phosphorylation. There was no effect of either subcutaneous or visceral adipose tissue EV on myotube insulin signaling. After exposure of hepatocyte cells to subcutaneous and visceral adipose tissue EV, there was a negative association between Akt signaling and glucose-6-phosphatase gene expression (24). Exosomes produced by visceral adipose tissue explants from obese humans can integrate into HepG2 cells and hepatic stellate cell lines (22). Gene expression profiling of HepG2 cells exposed to these exosomes indicated dysfunctional extracellular matrix regulation in HepG 2 cells and dysregulation of the TGF $\beta$ signaling pathways in both HepG2 and hepatic stellate cell lines (22). The authors proposed that in vivo these changes may induce liver fibrosis and may link obesity to non-alcoholic fatty liver disease (22). Similar observations were only made at high doses of visceral adipose tissue exosomes from a single lean individual but it is hard to draw conclusions regarding differences between exosomes from lean and obese individuals from this single experiment (22).
Adipocytes can be found in the tumor microenvironment and have been shown to promote tumor progression (34). Obesity is a risk factor for melanoma and its malignant progression. In a study of human subcutaneous adipocyte exosomes isolated by differential ultracentrifugation (35), there was a correlation between adipose tissue exosome shedding and donor BMI. When used at equal concentrations, exosomes from overweight and obese donors increased melanoma migration more than exosomes from lean individuals in a dose-dependent manner (35). This effect was thought to be mediated via fatty acid oxidation as inhibition by etomoxir reversed the effect (35). Interestingly, exosomes produced by mature 3T3-F442A adipocytes were found by mass spectrometry to contain an abundance of proteins involved in lipid metabolism, particularly those involved in fatty acid oxidation (35).

\section{Adipocyte EV and Whole Body Metabolic Status}

Circulating EV levels have been linked to cardiovascular disease (CVD), diabetes, and non-alcoholic fatty liver disease $(33,36,37)$ but the extent to which circulating EV derived from adipose tissue are involved is uncertain. Circulating EVs positive for CD9 and adiponectin have been isolated from the plasma of patients with aortic aneurysm (24) raising the possibility that adipose tissue may communicate with other organs via EV release. Obesity is a risk factor for CVD, type 2 diabetes and non-alcoholic fatty liver disease and may be associated with either higher EV release from adipose tissue stores or release of $\mathrm{EV}$ with a different functionality $(22,25,26,35)$ with downstream metabolic consequences for end organs involved in these diseases (Figure 1). The difficulty is being confident about which specific markers identify EV from adipose tissue. As discussed above, cellular experiments have suggested adiponectin, FABP-4, and RBP-4 to be adipocyte EV-specific markers. Proteomic profiling of extensively purified human plasma exosomes identified the presence of PPAR $\gamma$, a key transcription factor in adipocyte differentiation (38). However, since this protein can also be expressed in vascular endothelial cells and immune cells, this cannot categorically identify adipose tissue as a source of plasma EV. Similarly for adiponectin, it has been suggested that exosomes only account for a minor proportion of the total adiponectin secreted from 3T3-L1 cells (29) and, thus, it may not be a reliable marker for plasma EV. Others using mouse whole body models have suggested that perilipin A can act as an adipocyte EV marker $(32,39)$. The lack of definitive data on this issue makes it difficult to interpret experiments on circulating EV proposed to be from adipose tissue and the following data should be viewed with caution.

The number of visceral, but not subcutaneous, ex vivo adipose tissue-derived EV per gram of fat, quantified by flow cytometry, correlated with whole body HOMA-IR (but not body mass index or hsCRP levels) in patients $(n=11)$ with aortic aneurysm from whom the adipose tissue biopsies were collected (21). In a similar study, subcutaneous adipose tissue EVs produced from 16 individuals were inversely correlated with waist circumference and presence of metabolic syndrome, while visceral adipose tissue EV correlated positively with plasma liver enzymes (24). 
Circulating adipose microparticles, as determined by the presence of perilipin A, from ob/ob mice resulted in activation of monocytes in the circulation and in adipose tissue of wildtype mice (32). Perilipin A-positive EVs are higher in mice with diet-induced obesity and in humans with metabolic syndrome (39). In humans, circulating perilipin A-positive EV could be reduced by a 3 -month low calorie dietary intervention. Using FABP-4 as a marker of adipocyte-derived circulating exosomes, the change in adipocyte-derived exosome miRNA profile a year after gastric bypass surgery was assessed (40). Changes in miRNA profile that were predicted to regulate the insulin signaling pathway were observed and the degree of change in miRNA profile linked was correlated with both the change in IR as assessed by HOMA and the change in plasma levels of branched chain amino acids (40).

In a larger study of 1,012 patients with vascular disease, plasma $\mathrm{EV}$ were isolated by precipitation and characterized by multiplex immunoassay (41). Microvesicles containing the proteins cystatin C and CD14 have previously been shown to be associated with atheroma plaque size, and increased risk of CVD morbidity and mortality (42). The relationship between levels of these cystatin $\mathrm{C}$ positive and CD14-positive EV with subcutaneous and visceral adipose tissue thickness and metabolic disease prevalence and incidence was assessed. High EV-cystatin C levels were associated with high plasma hsCRP and low HDL-cholesterol (41). HDL-cholesterol was positively associated with EV-CD14 levels (41). EV-cystatin C levels were associated with increased odds of metabolic syndrome (OR 1.57; 95\% CI 1.19-2.17); however, there was no relationship between EV-cystatin-C levels and obesity or whole body IR (41). By contrast, EV-CD14 were associated with reduced hazard of developing type 2 diabetes (HR 0.84, 95\% CI 0.75-0.94) over a 6.5 years follow-up (41). Again it is difficult to know whether these EV risk markers are actually produced by adipose tissue. EV-cystatin C and EV-C14 are released by monocytes, endothelial cells, and platelets; but while CD14 adipose tissue gene expression and cystatin $\mathrm{C}$ protein secretion (43) is increased in adipose tissue from obese humans, there is no direct evidence that these proteins are secreted in EV from adipose tissue.

\section{ADIPOSE TISSUE-DERIVED MESENCHYMAL STEM CELLS (ADSC)}

There is great interest in adipose tissue as a source of stems cells for regenerative medicine for use in cancer and other diseases. Beneficial effects of such treatments may result from paracrine effects of ADSC mediated by EV. Exosomes produced by ADSC isolated from human adipose tissue have been shown to contain small RNA species predominately miRNA and snoRNA with some evidence that tRNA species are enriched (44). It appears that the contents do not merely reflect the source cellular content but that some RNA species are preferentially released (44). There is evidence that the protein secretory profile of ADSC differs from individual to individual and that raises the possibility that ADSC comprise a heterogeneous population of cells of functionally different subtypes and may produce corresponding EV (45). There are also data to suggest that functional aspects of ADSC differ from stem cells derived from other tissues (46). EVs from ADSC have been considered as a delivery route for delivering therapeutic miRNA to diseased cells.

\section{ADSC EV in Cancer}

Adipose tissue-derived mesenchymal stem cells were isolated from abdominal fat from patients undergoing neoplastic urological surgery and from participants without cancer (47). miRNA profiling of exosomes from these ADSC showed that, in both cancer and non-cancer patients, some exosome and ADSC miRNA content was similar. However, there did appear to be selective enrichment of some miRNA (let-7-a-1, miR-21, and miR-1260b) into exosomes (47). ADSC transfected with a miR-122 expression plasmid secreted exosomes containing miR-122. When the miR-122-containing exosomes were added to hepatocellular carcinoma cells, the cells became sensitive to chemotherapeutic drugs (48). Furthermore, intra-tumoural injection of transfected ADSC EV exosomes increased the effectiveness of an anti-cancer agent on hepatocellular carcinoma in an in vivo mouse model (48). ADSC exosomes have also been shown to promote migration of a breast cancer cell line (MCF7) (49).

\section{ADSC EV in Vascular Disease}

One study has compared EV produced by ADSC isolated from subcutaneous and visceral adipose tissue in obese and non-obese individuals (50). There was no difference in EV number or size between ADSC derived from non-obese or obese subjects (50). The content of VEGF and MMP-2 (markers of EV angiogenic potential) was lower in EV derived from the subcutaneous and the visceral compartment of obese individuals compared to those derived from the same compartments in non-obese individuals (50). In addition, the content of miR-126, but not that of miR-130A, (both pro-angiogenic miRs) was also reduced in EV produced by ADSC from obese individuals (50). These data suggesting that EV from obese ADSC, either from subcutaneous or from visceral depots, may have reduced pro-angiogenic potential were confirmed by in vitro experiments showing that EV from obese ADSC had reduced ability to induce migration and tube formation in cultured endothelial cells (50). Palmitic acid treatment could induce the anti-angiogenic EV changes in vitro and this suggests that increased circulating fatty acids in human obesity may influence the function of ADSC (50). ADSC EV secretion was stimulated by platelet-derived growth factor (PDGF) and changed EV protein composition enhancing their angiogenic properties (51). PDGF-stimulated ADSC were able to promote vessel formation when injected subcutaneously in Matrigel into mice (51). ADSC microvesicles have also been shown to increase migration and tube formation by human umbilical vein endothelial cells (52). The active agent carried in the microvesicles was thought to be miR-31 that inhibited factorinhibiting HIF-1 (52).

Restenosis of vein grafts is a particular problem with coronary artery bypass surgery. ADSC have been used to try and reduce neointimal hyperplasia in vein grafts. Human ADSC were shown, by in vitro cell culture and in a mouse model, to reduce vein graft neointima formation by inhibiting vascular smooth muscle cell proliferation and migration and reducing macrophage 
migration and inflammation (53). ADSC also inhibit the activation, differentiation, and proliferation of $\mathrm{T}$ cells (54). In a porcine model of metabolic syndrome and renal artery stenosis, treatment with autologous EV from ADSC resulted in decreased renal inflammation and increased kidney blood flow and glomerular filtration rate (55). Others have found that a combined treatment of ADSC plus ADSC-derived exosomes had superior ability, compared to either one alone, to protect the kidney from acute ischemia-reperfusion injury in a rat model (56).

\section{ADSC and Neurodegenerative Disease}

ADSC are also of interest for the treatment of neurodegenerative disorders such as amyotrophic lateral sclerosis (57). Murine ADSC exosomes protect motor neurone-like NSC-34 cells from oxidative damage increasing their viability (57) and similarly human ADSC exosomes protect neurones against glutamate-induced damage (58). Others have shown in mice in vitro that ADSC nanovesicles and microvesicles prevented apoptosis in neuronal cells and increased remyelination in cerebellar slices demyelinated using lysophosphatidyl choline (59). Again EV have been considered as a delivery vehicle with therapeutic implications for neurodegenerative disease. ADSC secreted exosomes containing neprilysin, a major $\beta$-amyloid peptide degrading enzyme, and the exosomes were capable of delivering this protein to a neuroblastoma cell line (N2a) resulting in decreased secreted and intracellular $\beta$-amyloid peptide (60). Therefore, there is therapeutic potential for such EV in Alzheimer's disease. Furthermore, exosomes from ADSC have been shown to have therapeutic potential in an in vitro mouse neuronal cell model of Huntington's disease (61).

\section{CONCLUSION}

Adipose tissue can produce EV from both adipocytes and ADSC (Figure 1). The early data presented above indicate that the

\section{REFERENCES}

1. Greenberg AS, McDaniel ML. Identifying the links between obesity, insulin resistance and beta-cell function: potential role of adipocyte-derived cytokines in the pathogenesis of type 2 diabetes. Eur J Clin Invest (2002) 32(Suppl 3): 24-34. doi:10.1046/j.1365-2362.32.s3.4.x

2. Rosen ED, Spiegelman BM. Adipocytes as regulators of energy balance and glucose homeostasis. Nature (2006) 444:847-53. doi:10.1038/nature05483

3. Gho YS, Lee C. Emergent properties of extracellular vesicles: a holistic approach to decode the complexity of intercellular communication networks. Mol Biosyst (2017) 13:1291-6. doi:10.1039/c7mb00146k

4. Borges FT, Reis LA, Schor N. Extracellular vesicles: structure, function, and potential clinical uses in renal diseases. Braz J Med Biol Res (2013) 46:824-30. doi:10.1590/1414-431X20132964

5. Momen-Heravi F, Balaj L, Alian S, Mantel PY, Halleck AE, Trachtenberg AJ, et al. Current methods for the isolation of extracellular vesicles. Biol Chem (2013) 394:1253-62. doi:10.1515/hsz-2013-0141

6. Huang-Doran I, Zhang CY, Vidal-Puig A. Extracellular vesicles: novel mediators of cell communication in metabolic disease. Trends Endocrinol Metab (2017) 28:3-18. doi:10.1016/j.tem.2016.10.003

7. Booth A, Magnuson A, Foster M. Detrimental and protective fat: body fat distribution and its relation to metabolic disease. Horm Mol Biol Clin Investig (2014) 17:13-27. doi:10.1515/hmbci-2014-0009

8. Ibrahim MM. Subcutaneous and visceral adipose tissue: structural and functional differences. Obes Rev (2010) 11:11-8. doi:10.1111/j.1467-789X. 2009.00623.x adipose depot from which adipocyte EV are derived, and the relative state of obesity of the donor may influence the function of such EV. We are still at the first stages of research. No definitive marker of adipocyte-derived EV has yet been agreed upon nor has a consensus characteristic EV cargo been identified. This makes it difficult to interpret data on circulating EV in obesity and metabolic diseases such as type 2 diabetes. Although the data do suggest that circulating EV profiles change in obesity and metabolic disease, it is not yet clear whether EVs act merely as a marker of disease or whether they play a functional role in tissue to tissue communication. Initial cell culture and animal model experiments do suggest that there is the potential for communication between adipocytes and immune and liver cells via EV. Adipose tissue has also proved to be a source of stem cells and EV produced by ADSC appear to have potential roles in cancer, angiogenesis, vascular disease, and neurodegenerative disease, both as a direct effector to promote or prevent pathogenesis and as a delivery system to target beneficial miRNA to cells as a therapeutic option.

\section{AUTHOR CONTRIBUTIONS}

$\mathrm{XG}$ reviewed the literature, wrote the first draft, and created the figure. CS reviewed the article for accuracy as EV expert and edited final draft. DF reviewed the literature, wrote the final draft, and made editorial changes.

\section{ACKNOWLEDGMENTS}

We gratefully acknowledge funding from the following sources: Diabetes UK 15/0005182, Lions Medical Research Foundation (LMRF), The University of Queensland, and Fondo Nacional de Desarrollo Científico y Tecnológico (FONDECYT 1170809).

9. Jensen MD. Role of body fat distribution and the metabolic complications of obesity. JClin Endocrinol Metab (2008) 93:S57-63. doi:10.1210/jc. 2008-1585

10. Mathieu P, Boulanger MC, Despres JP. Ectopic visceral fat: a clinical and molecular perspective on the cardiometabolic risk. Rev Endocr Metab Disord (2014) 15:289-98. doi:10.1007/s11154-014-9299-3

11. van Marken Lichtenbelt WD, Vanhommerig JW, Smulders NM, Drossaerts JM, Kemerink GJ, Bouvy ND, et al. Cold-activated brown adipose tissue in healthy men. N Engl JMed (2009) 360:1500-8. doi:10.1056/NEJMoa 0808718

12. Trayhurn P. Origins and early development of the concept that brown adipose tissue thermogenesis is linked to energy balance and obesity. Biochimie (2017) 134:62-70. doi:10.1016/j.biochi.2016.09.007

13. Harms M, Seale P. Brown and beige fat: development, function and therapeutic potential. Nat Med (2013) 19:1252-63. doi:10.1038/nm.3361

14. Large V, Peroni O, Letexier D, Ray H, Beylot M. Metabolism of lipids in human white adipocyte. Diabetes Metab (2004) 30:294-309. doi:10.1016/ S1262-3636(07)70121-0

15. Gimble JM, Bunnell BA, Frazier T, Rowan B, Shah F, Thomas-Porch C, et al. Adipose-derived stromal/stem cells: a primer. Organogenesis (2013) 9:3-10. doi:10.4161/org.24279

16. Baer PC, Geiger H. Adipose-derived mesenchymal stromal/stem cells: tissue localization, characterization, and heterogeneity. Stem Cells Int (2012) 2012:812693. doi:10.1155/2012/812693

17. Arner P, Spalding KL. Fat cell turnover in humans. Biochem Biophys Res Commun (2010) 396:101-4. doi:10.1016/j.bbrc.2010.02.165 
18. Cristancho AG, Lazar MA. Forming functional fat: a growing understanding of adipocyte differentiation. Nat Rev Mol Cell Biol (2011) 12:722-34. doi:10.1038/nrm3198

19. Cuthbertson DJ, Steele T, Wilding JP, Halford JC, Harrold JA, Hamer M, et al. What have human experimental overfeeding studies taught us about adipose tissue expansion and susceptibility to obesity and metabolic complications? Int J Obes (Lond) (2017) 41(6):853-65. doi:10.1038/ijo.2017.4

20. Sattar N, Gill JM. Type 2 diabetes in migrant south Asians: mechanisms, mitigation, and management. Lancet Diabetes Endocrinol (2015) 3:1004-16. doi:10.1016/S2213-8587(15)00326-5

21. Kranendonk ME, Visseren FL, van Balkom BW, Nolte-'t Hoen EN, van Herwaarden JA, de Jager W, et al. Human adipocyte extracellular vesicles in reciprocal signaling between adipocytes and macrophages. Obesity (Silver Spring) (2014) 22:1296-308. doi:10.1002/oby.20679

22. Koeck ES, Iordanskaia T, Sevilla S, Ferrante SC, Hubal MJ, Freishtat RJ, et al. Adipocyte exosomes induce transforming growth factor beta pathway dysregulation in hepatocytes: a novel paradigm for obesity-related liver disease. J Surg Res (2014) 192:268-75. doi:10.1016/j.jss.2014.06.050

23. Fischer-Posovszky P, Newell FS, Wabitsch M, Tornqvist HE. Human SGBS cells - a unique tool for studies of human fat cell biology. Obes Facts (2008) 1:184-9. doi:10.1159/000145784

24. KranendonkME, VisserenFL, van HerwaardenJA,Nolte-'tHoenEN, deJagerW, Wauben $\mathrm{MH}$, et al. Effect of extracellular vesicles of human adipose tissue on insulin signaling in liver and muscle cells. Obesity (Silver Spring) (2014) 22:2216-23. doi:10.1002/oby.20847

25. Ferrante SC, Nadler EP, Pillai DK, Hubal MJ, Wang Z, Wang JM, et al. Adipocyte-derived exosomal miRNAs: a novel mechanism for obesity-related disease. Pediatr Res (2015) 77:447-54. doi:10.1038/pr.2014.202

26. Lee JE, Moon PG, Lee IK, Baek MC. Proteomic analysis of extracellular vesicles released by adipocytes of Otsuka Long-Evans Tokushima Fatty (OLETF) rats. Protein J (2015) 34:220-35. doi:10.1007/s10930-015-9616-z

27. Connolly KD, Guschina IA, Yeung V, Clayton A, Draman MS, Von Ruhland C, et al. Characterisation of adipocyte-derived extracellular vesicles released pre- and post-adipogenesis. J Extracell Vesicles (2015) 4:29159. doi:10.3402/ jev.v4.29159

28. Komai AM, Brannmark C, Musovic S, Olofsson CS. PKA-independent cAMP stimulation of white adipocyte exocytosis and adipokine secretion: modulations by Ca2+ and ATP. J Physiol (2014) 592:5169-86. doi:10.1113/ jphysiol.2014.280388

29. DeClercq V, d'Eon B, McLeod RS. Fatty acids increase adiponectin secretion through both classical and exosome pathways. Biochim Biophys Acta (2015) 1851:1123-33. doi:10.1016/j.bbalip.2015.04.005

30. Sano S, Izumi Y, Yamaguchi T, Yamazaki T, Tanaka M, Shiota M, et al. Lipid synthesis is promoted by hypoxic adipocyte-derived exosomes in 3T3-L1 cells. Biochem Biophys Res Commun (2014) 445:327-33. doi:10.1016/j. bbrc.2014.01.183

31. Chen Y, Buyel JJ, Hanssen MJ, Siegel F, Pan R, Naumann J, et al. Exosomal microRNA miR-92a concentration in serum reflects human brown fat activity. Nat Commun (2016) 7:11420. doi:10.1038/ncomms11420

32. Eguchi A, Mulya A, Lazic M, Radhakrishnan D, Berk MP, Povero D, et al. Microparticles release by adipocytes act as "find-me" signals to promote macrophage migration. PLoS One (2015) 10:e0123110. doi:10.1371/journal. pone. 0123110

33. Deng ZB, Poliakov A, Hardy RW, Clements R, Liu C, Liu Y, et al. Adipose tissue exosome-like vesicles mediate activation of macrophage-induced insulin resistance. Diabetes (2009) 58:2498-505. doi:10.2337/db09-0216

34. Dirat B, Bochet L, Escourrou G, Valet P, Muller C. Unraveling the obesity and breast cancer links: a role for cancer-associated adipocytes? Endocr Dev (2010) 19:45-52. doi:10.1159/000316896

35. Lazar I, Clement E, Dauvillier S, Milhas D, Ducoux-Petit M, LeGonidec S, et al. Adipocyte exosomes promote melanoma aggressiveness through fatty acid oxidation: a novel mechanism linking obesity and cancer. Cancer Res (2016) 76:4051-7. doi:10.1158/0008-5472.CAN-16-0651

36. Ban LA, Shackel NA, McLennan SV. Extracellular vesicles: a new frontier in biomarker discovery for non-alcoholic fatty liver disease. Int J Mol Sci (2016) 17:376. doi:10.3390/ijms17030376

37. Bank IE, Timmers L, Gijsberts CM, Zhang YN, Mosterd A, Wang JW, et al. The diagnostic and prognostic potential of plasma extracellular vesicles for cardiovascular disease. Expert Rev Mol Diagn (2015) 15:1577-88. doi:10.158 6/14737159.2015.1109450

38. Looze C, Yui D, Leung L, Ingham M, Kaler M, Yao X, et al. Proteomic profiling of human plasma exosomes identifies PPARgamma as an exosome-associated protein. Biochem Biophys Res Commun (2009) 378:433-8. doi:10.1016/j. bbrc.2008.11.050

39. Eguchi A, Lazic M, Armando AM, Phillips SA, Katebian R, Maraka S, et al. Circulating adipocyte-derived extracellular vesicles are novel markers of metabolic stress. JMol Med (Berl) (2016) 94:1241-53. doi:10.1007/ s00109-016-1446-8

40. Hubal MJ, Nadler EP, Ferrante SC, Barberio MD, Suh JH, Wang J, et al. Circulating adipocyte-derived exosomal microRNAs associated with decreased insulin resistance after gastric bypass. Obesity (Silver Spring) (2017) 25:102-10. doi:10.1002/oby.21709

41. Kranendonk ME, de Kleijn DP, Kalkhoven E, Kanhai DA, Uiterwaal CS, van der Graaf Y, et al. Extracellular vesicle markers in relation to obesity and metabolic complications in patients with manifest cardiovascular disease. Cardiovasc Diabetol (2014) 13:37. doi:10.1186/1475-2840-13-37

42. Kanhai DA, Visseren FL, van der Graaf Y, Schoneveld AH, Catanzariti LM, Timmers L, et al. Microvesicle protein levels are associated with increased risk for future vascular events and mortality in patients with clinically manifest vascular disease. Int J Cardiol (2013) 168:2358-63. doi:10.1016/j. ijcard.2013.01.231

43. Naour N, Fellahi S, Renucci JF, Poitou C, Rouault C, Basdevant A, et al. Potential contribution of adipose tissue to elevated serum cystatin $\mathrm{C}$ in human obesity. Obesity (Silver Spring) (2009) 17:2121-6. doi:10.1038/oby.2009.96

44. Baglio SR, Rooijers K, Koppers-Lalic D, Verweij FJ, Perez Lanzon M, Zini N, et al. Human bone marrow- and adipose-mesenchymal stem cells secrete exosomes enriched in distinctive miRNA and tRNA species. Stem Cell Res Ther (2015) 6:127. doi:10.1186/s13287-015-0116-Z

45. Kalinina N, Kharlampieva D, Loguinova M, Butenko I, Pobeguts O, Efimenko A, et al. Characterization of secretomes provides evidence for adipose-derived mesenchymal stromal cells subtypes. Stem Cell Res Ther (2015) 6:221. doi:10.1186/s13287-015-0209-8

46. Del Fattore A, Luciano R, Saracino R, Battafarano G, Rizzo C, Pascucci L, et al. Differential effects of extracellular vesicles secreted by mesenchymal stem cells from different sources on glioblastoma cells. Expert Opin Biol Ther (2015) 15:495-504. doi:10.1517/14712598.2015.997706

47. Garcia-Contreras M, Vera-Donoso CD, Hernandez-Andreu JM, GarciaVerdugo JM, Oltra E. Therapeutic potential of human adipose-derived stem cells (ADSCs) from cancer patients: a pilot study. PLoS One (2014) 9:e113288. doi:10.1371/journal.pone.0113288

48. Lou G, Song X, Yang F, Wu S, Wang J, Chen Z, et al. Exosomes derived from miR-122-modified adipose tissue-derived MSCs increase chemosensitivity of hepatocellular carcinoma. J Hematol Oncol (2015) 8:122. doi:10.1186/ s13045-015-0220-7

49. Lin R, Wang S, Zhao RC. Exosomes from human adipose-derived mesenchymal stem cells promote migration through Wnt signaling pathway in a breast cancer cell model. Mol Cell Biochem (2013) 383:13-20. doi:10.1007/ s11010-013-1746-z

50. Togliatto G, Dentelli P, Gili M, Gallo S, Deregibus C, Biglieri E, et al. Obesity reduces the pro-angiogenic potential of adipose tissue stem cell-derived extracellular vesicles (EVs) by impairing miR-126 content: impact on clinical applications. Int J Obes (Lond) (2016) 40(1):102-11. doi:10.1038/ijo.2015.123

51. Lopatina T, Bruno S, Tetta C, Kalinina N, Porta M, Camussi G. Platelet-derived growth factor regulates the secretion of extracellular vesicles by adipose mesenchymal stem cells and enhances their angiogenic potential. Cell Commun Signal (2014) 12:26. doi:10.1186/1478-811X-12-26

52. Kang T, Jones TM, Naddell C, Bacanamwo M, Calvert JW, Thompson WE, et al. Adipose-derived stem cells induce angiogenesis via microvesicle transport of miRNA-31. Stem Cells Transl Med (2016) 5:440-50. doi:10.5966/ sctm.2015-0177

53. Liu R, Shen H, Ma J, Sun L, Wei M. Extracellular vesicles derived from adipose mesenchymal stem cells regulate the phenotype of smooth muscle cells to limit intimal hyperplasia. Cardiovasc Drugs Ther (2016) 30:111-8. doi:10.1007/ s10557-015-6630-5

54. Blazquez R, Sanchez-Margallo FM, de la Rosa O, Dalemans W, Alvarez V, Tarazona $\mathrm{R}$, et al. Immunomodulatory potential of human adipose 
mesenchymal stem cells derived exosomes on in vitro stimulated T cells. Front Immunol (2014) 5:556. doi:10.3389/fimmu.2014.00556

55. Eirin A, Zhu XY, Puranik AS, Tang H, McGurren KA, van Wijnen AJ, et al. Mesenchymal stem cell-derived extracellular vesicles attenuate kidney inflammation. Kidney Int (2017) 92(1):114-24. doi:10.1016/j.kint.2016.12.023

56. Lin KC, Yip HK, Shao PL, Wu SC, Chen KH, Chen YT, et al. Combination of adipose-derived mesenchymal stem cells (ADMSC) and ADMSC-derived exosomes for protecting kidney from acute ischemia-reperfusion injury. Int J Cardiol (2016) 216:173-85. doi:10.1016/j.ijcard.2016.04.061

57. Bonafede R, Scambi I, Peroni D, Potrich V, Boschi F, Benati D, et al. Exosome derived from murine adipose-derived stromal cells: neuroprotective effect on in vitro model of amyotrophic lateral sclerosis. Exp Cell Res (2016) 340:150-8. doi:10.1016/j.yexcr.2015.12.009

58. Wei JJ, Chen YF, Xue CL, Ma BT, Shen YM, Guan J, et al. Protection of nerve injury with exosome extracted from mesenchymal stem cell. Zhongguo Yi Xue Ke Xue Yuan Xue Bao (2016) 38:33-6. doi:10.3881/j.issn.1000-503X.2016. 01.006

59. Farinazzo A, Turano E, Marconi S, Bistaffa E, Bazzoli E, Bonetti B. Murine adipose-derived mesenchymal stromal cell vesicles: in vitro clues for neuroprotective and neuroregenerative approaches. Cytotherapy (2015) 17:571-8. doi:10.1016/j.jcyt.2015.01.005

60. Katsuda T, Tsuchiya R, Kosaka N, Yoshioka Y, Takagaki K, Oki K, et al. Human adipose tissue-derived mesenchymal stem cells secrete functional neprilysin-bound exosomes. Sci Rep (2013) 3:1197. doi:10.1038/srep01197

61. Lee M, Liu T, Im W, Kim M. Exosomes from adipose-derived stem cells ameliorate phenotype of Huntington's disease in vitro model. Eur J Neurosci (2016) 44:2114-9. doi:10.1111/ejn.13275

Conflict of Interest Statement: The authors declare that the research was conducted in the absence of any commercial or financial relationships that could be construed as a potential conflict of interest.

Copyright $\odot 2017$ Gao, Salomon and Freeman. This is an open-access article distributed under the terms of the Creative Commons Attribution License (CC BY). The use, distribution or reproduction in other forums is permitted, provided the original author(s) or licensor are credited and that the original publication in this journal is cited, in accordance with accepted academic practice. No use, distribution or reproduction is permitted which does not comply with these terms. 